\title{
Essais
}

Revue interdisciplinaire d'Humanités

Hors-série 7 | 2022

Récits d'outre-thèse

\section{Un diplôme pour la gloire? La reconnaissance du titre de docteur, un parcours du combattant}

L'art d'être et paraître au monde ou l'anneau de Saturne

A degree for glory? The recognition of the title of doctor: A battlefield The art of being and appearing in the world or the ring of Saturn

\section{Damien Plantey}

\section{OpenEdition}

Journals

Édition électronique

URL : https://journals.openedition.org/essais/10825

DOI : 10.4000/essais. 10825

ISSN : 2276-0970

Éditeur

École doctorale Montaigne Humanités

Édition imprimée

Date de publication : 15 avril 2022

ISBN : 979-10-970024-00-0

ISSN : $2417-4211$

Référence électronique

Damien Plantey, « Un diplôme pour la gloire ? La reconnaissance du titre de docteur, un parcours du combattant », Essais [En ligne], Hors-série 7 | 2022, mis en ligne le 01 février 2022, consulté le 18 janvier 2023. URL : http://journals.openedition.org/essais/10825 ; DOI : https://doi.org/10.4000/ essais. 10825

Ce document a été généré automatiquement le 18 janvier 2023.

Tous droits réservés 


\title{
Un diplôme pour la gloire? La reconnaissance du titre de docteur, un parcours du combattant
}

\author{
L'art d'être et paraître au monde ou l'anneau de Saturne
}

A degree for glory? The recognition of the title of doctor: A battlefield The art of being and appearing in the world or the ring of Saturn

Damien Plantey

1 Docteur en histoire, j'ai soutenu ma thèse le 22 janvier 2011 à l'Université Bordeaux Montaigne. J'ai obtenu le diplôme de docteur en histoire moderne et contemporaine mention très honorable avec les félicitations.

Mon inscription en thèse sous la direction de Madame Anne-Marie Cocula remontait à plus d'une décennie auparavant, en octobre de l'année universitaire 1999-2000. Enseignant en histoire-géographie je m'étais lancé dans un double projet pour mener en parallèle ma reconversion professionnelle dans les bibliothèques de l'Enseignement supérieur et de la recherche et ma thèse de doctorat sur l'histoire des bibliothèques à la Renaissance. Je savais que mon projet de recherche serait à organiser sur un temps libre déjà très occupé par mon projet de reconversion professionnelle pour devenir bibliothécaire. De sorte que ma thèse était d'emblée placée sous le signe du long terme, et de la ténacité. Je ne me doutais pas qu'après l'obtention de mon titre de docteur, un véritable parcours du combattant commencerait pour sa reconnaissance par la société.

2 Mon projet de thèse faisait suite à des recherches et un mémoire pour le diplôme d'études approfondies en histoire, portant sur la diffusion des idées religieuses nouvelles par des femmes de la noblesse française aux débuts de la Réforme au $\mathrm{XVI}^{\mathrm{e}}$ siècle. Ma thèse de doctorat a prolongé l'étude des idées et des goûts développés de mère en fille par trois de ces femmes à travers la reconstitution de leur bibliothèque, Marguerite de Navarre, Jeanne d'Albret et Catherine de Bourbon. Le caractère inédit de la thèse repose sur la reconstitution de la bibliothèque que ces trois princesses lettrées se sont transmise, participant à l'essor du modèle de la bibliothèque universaliste à la 
Renaissance. La problématique porte sur la question de la reconstitution d'une bibliothèque, alors qu'aucun exemplaire n'a été conservé, en ne pouvant compter que sur des inventaires et des comptes (les sources traditionnelles de l'histoire des bibliothèques) lacunaires et sans mention de titres. Il s'agissait par conséquent de reconstituer la bibliothèque sans les livres.

3 En ce qui concerne mon projet de reconversion professionnelle entrepris au moment de mon inscription en thèse, après un premier emploi en bibliothèque de recherche (une mise à l'étrier) j'ai été recruté au Service commun de documentation de l'Université Bordeaux Montaigne en tant que bibliothécaire. J'ai suivi une formation interne approfondie et j'ai travaillé au Centre d'acquisition et de diffusion de l'information scientifique et technique à la bibliothèque universitaire en sciences humaines ainsi qu'à la bibliothèque Élie Vinet en histoire et histoire de l'art. Mon expérience de la recherche documentaire en tant que doctorant m'a aidé à développer les compétences professionnelles dans les renseignements et la recherche bibliographiques propres au métier de bibliothécaire. De sorte que mes expériences en tant que doctorant et bibliothécaire se sont complétées, me donnant une double culture du travail en bibliothèque comme chercheur et professionnel.

4 L'obtention du concours de bibliothécaire d'État à l'École nationale supérieure des sciences de l'information et des bibliothèques (Enssib) m'a permis de stabiliser ma situation professionnelle. Durant la préparation de ce concours, effectuée en parallèle de mes premières années de recherche en tant que doctorant, le temps libre que j'ai pu consacrer à ma thèse a été très contraint et restreint. J'ai avancé très lentement sur la définition de mon sujet et sa délimitation ainsi que sur la constitution de mon corpus de sources, d'autant plus que ledit corpus s'est avéré être conservé principalement aux Archives départementales des Pyrénées atlantiques à Pau et que je vivais et travaillais à Bordeaux.

5 Cette mise à distance imposée d'un espace-temps dans mon travail de recherche consacré au dépouillement et à l'analyse des registres d'archives a donné lieu à une méthode de travail impliquant une mise en perspective toujours renouvelée de mon corpus de sources, ce que j'ai appelé dans mon introduction lors de ma soutenance de thèse la "mise en contexte" du corpus. Or cette méthodologie s'est avérée non seulement d'une grande justesse pour la modélisation de ma thèse mais également très précieuse dans la préparation du concours national de bibliothécaire menée durant ces premières années de thèse. Grande justesse qui a donné sa qualité inédite et sa pertinence reconnue à ma thèse dont le propos élargit le champ des sources de l'histoire des bibliothèques. Une méthode qui a été très précieuse dans l'analyse des sujets et des textes des épreuves écrites et orales du concours de bibliothécaire, autour des thématiques inscrites à son programme comme la bibliographie, l'historiographie et l'épistémologie.

6 Intitulée «La bibliothèque utile : la bibliothèque, le silence et la nuit », la troisième partie de ma thèse, portant sur l'étude de l'espace et des usages de la bibliothèque, n'aurait pas existé sans cette mise en œuvre méthodologique de mise à distance et en contexte des sources. La mise à distance méthodologique a permis la mise en perspective synthétique. Or ladite partie a été reconnue par le jury comme la plus originale et démonstrative en ce sens que j'y développe une analyse de la «profondeur » de la bibliothèque. Comme les «armoires faictes aux recoins ${ }^{1}$ » et aux portes dérobées derrière les décors en trompe-l'œil «à moresques » peints et vernis, 
dissimulant les étagères coulissantes et pivotantes et les tiroirs à double fond dans les cabinets d'étude où Catherine de Bourbon la sœur d'Henri IV conservait ses livres censurés et ses bijoux talismans au pouvoir magique, toutes collections ainsi mises au secret, les sources écrites pour être analysées doivent s'apprécier dans toute leur profondeur. Autrement dit, la bibliothèque doit être étudiée dans tout son contexte, dans ses moindres recoins, comme le suggère le sous-titre du livre issu de ma thèse réécrite et publiée aux Presses de l'Enssib: Les bibliothèques des princesses de Navarre au $X V I^{e}$ siècle : livres, objets, mobilier, décor, espaces et usages.

7 Ma démarche épistémologique avait pour objectif de montrer que la bibliothèque était bien plus qu'une liste de titres de livres. Lors de la soutenance un membre du jury a commencé son intervention en se remémorant la perplexité dubitative avec laquelle elle avait accueilli mon projet de thèse à la suite de notre première entrevue au début de mes recherches: reconstituer une bibliothèque sans trace apparente aucune des livres qui la composaient! Le premier conseil donné par ma directrice de thèse, choisir et construire un sujet passionnant, "un sujet qui vous tienne à cœur ", ainsi que sa disponibilité motivante durant toutes ces années, m'ont permis de persévérer dans mes recherches. Je mesure également aujourd'hui combien le temps long imposé au dépouillement, à l'analyse et à la synthèse, a favorisé le recul et la mise en perspective nécessaires.

8 Après l'obtention du concours de bibliothécaire, le travail de rédaction et de finalisation de la thèse s'est effectué au gré de mes affectations successives en région parisienne et à Paris, à la Bibliothèque nationale de France où j'ai intégré le département spécialisé des Estampes et de la photographie pour devenir bibliothécaire gestionnaire de la collection de photographie contemporaine. Ma fonction de bibliothécaire m'a permis d'acquérir des compétences dans la valorisation des collections patrimoniales notamment à travers la participation à l'organisation d'expositions m'offrant l'opportunité de nombreuses missions effectuées en France et à l'étranger dans des institutions culturelles diversifiées. Si l'articulation de mon expérience professionnelle en tant que bibliothécaire de la collection de photographie contemporaine de la BnF avec mon projet de recherche en tant que doctorant sur l'histoire des bibliothèques à la Renaissance peut paraître inexistante, à l'image de deux lignes d'horizon parallèles et superposées, il faut au contraire avoir à l'esprit que deux lignes parallèles suivent la même direction et peuvent finir par se croiser. Travail et recherche n'ont jamais été autant liés, l'expression travail de recherche jamais autant justifiée.

9 Lors de ma soutenance un membre du jury a relevé le peu de références bibliographiques accessibles en ligne ce à quoi j'ai répondu que j'avais commencé ma thèse « en 1999 c'est-à-dire au siècle dernier ! » tout en soulignant que les références numériques balbutiaient encore en ce début de décennie 2010. Cela avait par conséquent impliqué un travail de dépouillement des sources effectué exclusivement sur place en archives et intra-muros en bibliothèque.

10 Après mon déménagement à Paris avec l'enjeu professionnel de ma titularisation, le fil de mes recherches a été difficile à retisser. J'avais alors néanmoins l'acquis de l'identification et de la délimitation de mon corpus de sources et décidé avec ma directrice de thèse d'une nouvelle mise en perspective de mon sujet qui s'est avéré fonder la qualité inédite de ma thèse : donner à voir l'histoire des bibliothèques au travers non seulement des livres mais également de leur «contexte» mobilier et 
décoratif, spatial et d'usage. J'ai repris le fastidieux dépouillement des sources, tel un rat de bibliothèque et d'archives, durant des séjours réguliers à Pau précédés et terminés par de longs trajets entre Paris et le Béarn. Dix années plus tard, ma thèse soutenue, invité aux Conférences d'Orion près d'Orthez en Béarn pour échanger avec François Bayrou et Jean-Marc Terrasse sur mon livre (ma thèse publiée), j'ai eu l'occasion de me remémorer à haute voix ces longs trajets pour gagner le Béarn depuis Paris, d'autant plus que ce jour-là le trajet avait duré onze heures, pour cause de panne de TGV, sous la canicule, si bien que j'étais arrivé sur place (épuisé) dix minutes avant de monter sur l'estrade. J'ai alors fait le parallèle entre mon " périple» du jour et les longues semaines effectuées par les reines de Navarre mère et fille Marguerite et Jeanne d'Albret dans leurs voyages incessants de vie itinérante harassante entre la cour de France et la cour de Pau, la première écrivant ses célèbres contes, bercée dans sa litière à dos de mulets, la deuxième des épîtres poétiques installée dans son charriot muni d'un poêle à charbon. La bibliothèque était itinérante et dimensionnée par le voyage au long cours au XVI ${ }^{e}$ siècle... et encore en ce début de XXI siècle! Mais ce périple dans le parcours de valorisation de ma thèse publiée auprès d'un public amateur d'histoire était une péripétie anecdotique en comparaison des obstacles à franchir pour la reconnaissance de mon titre de docteur par la société, à commencer précisément par le monde de l'édition.

11 Après l'obtention du doctorat et deux longues années de travail à la recherche d'un éditeur, le Comité éditorial des Presses de l'Enssib a sélectionné mon projet de publication de thèse. J'ai par la suite eu l'opportunité de faire la présentation de ma thèse et de mon livre ${ }^{2}$ devant un auditoire composé de chercheurs, de doctorants et de professionnels des bibliothèques dans le cadre d'un atelier de recherche universitaire à l'Institut national d'histoire de l'art (INHA) où j'exerce maintenant mon métier de bibliothécaire depuis plusieurs années. S'il s'agissait bien d'une forme de reconnaissance de mes compétences scientifiques dans un cadre professionnel, cependant, force est de constater que mon diplôme de doctorat «bac+8 " n'est pas reconnu stricto sensu à titre professionnel, la condition de diplôme exigée pour concourir à la fonction de bibliothécaire étant la licence « bac+3 ».

12 À défaut de reconnaissance professionnelle, mon titre de docteur me donne la perspective d'une reconnaissance intellectuelle. Mon livre (ma thèse réécrite et publiée) m'a donné la satisfaction personnelle d'avoir servi de source d'inspiration dans l'élaboration du décor éphémère d'un défilé de haute couture parisien (pour présenter sur un mode visuel inspiré, habillé, une mode habitée par l'histoire de l'art des arts décoratifs au féminin). La revue de l'INHA Perspectives consacre en 2021 un numéro sur l'étude des "manières d'habiter et d'être habité ». J'ai fait la proposition d'un article intitulé « La bibliothèque habitée au féminin à la Renaissance : l'art d'être et paraitre au monde ». Une manière de poursuivre le parcours pour la reconnaissance de mon titre de docteur et de ne pas me résigner à un diplôme pour la gloire?

13 La clé de la reconnaissance est en effet à mon sens à rechercher dans le prolongement et le renouvellement des activités de recherche de manière à mettre la thèse en résonance avec la société, c'est pour le docteur l'art et la manière d'être et paraître au monde. Marguerite de Navarre conservait précieusement la clé de son grand cabinet de bibliothèque contenant ses cadrans astrologiques lunaires et solaires, le coffre-fort dans lequel sa fille Jeanne d'Albret a elle-même conservé ses livres de psaumes mis à l'Index et ses livres de recettes secrètes de poisons et leurs remèdes antidotes, un 
corpus écrit ô combien précieux dans l'art de cheminer corps et âme de par le monde, l'art d'être et paraître au monde. Dans sa Comédie de Mont-de-Marsan Marguerite de Navarre questionne l'être et le paraître ${ }^{3}$, le jeu des rôles et des masques sur la scène du monde. Placée par sa naissance sous l'auspice zodiacal de la planète Saturne, la reine prenait conseil auprès du cercle formé autour d'elle par ses astrologues se servant de ses cadrans, précieux instruments de reconnaissance pour établir la carte du ciel et voir juste. Si ma thèse aboutit à la reconstitution d'une bibliothèque sans les livres, sans la reconnaissance de la société mon titre de docteur représente une forme d'inachevé, Saturne sans son anneau. Le titre de docteur a besoin de la reconnaissance de la société pour former un tout abouti.

\section{NOTES}

1. D. Plantey Les bibliothèques des princesses de Navarre au XVIe siècle: livres, objets, mobilier, décor, espaces et usages, Presses de l'Enssib, 2016, p. 70

2. D. Plantey, Les bibliothèques des princesses de Navarre au XVI siècle: livres, objets, mobilier, décor, espaces et usages, op.cit.

3. Théâtre de femmes sous l'Ancien Régime, éd. A. Evain, P. Gethner, H. Goldwyn, vol. 1 (XVI siècle), Publications de l'Université de Saint-Étienne (La cité des dames), 2006, p. 202-229.

\section{AUTEUR}

\section{DAMIEN PLANTEY}

Bibliothécaire à l'Institut national d'histoire de l'art 